\title{
Dynamics of rubber band stretch ejection
}

\author{
Xiang Li*) Bohua Sun*,1) Yi Zhang*) Yuanfan Dai*)1 \\ 1*(School of Civil Engineering 83 Institute of Mechanics and Technology, \\ Xi'an University of Architecture and Technology, Xi'an 710055, China)
}

\begin{abstract}
Why do stretched rubber bands not hit the hand after ejection? What is the mechanism behind the rubber band ejection dynamics? These questions represent a fascinating scientific problem. Because the size of a rubber band in the circumferential direction is much larger than that in the other two directions of its cross-section, we regard the rubber band as a slender beam and establish a mathematical model of the dynamics of the rubber band stretching and ejection. Furthermore, we obtain the dependence of the dynamic curvature of the rubber band on the arc length and time. We used the finite element software ABAQUS to simulate the dynamic process of a rubber band stretching and ejection. The simulation results and dimensional analysis were performed to examine the effect of the bending elastic rebound velocity. The mathematical model and simulation results revealed that the relationship between the curvature and time at the end of the rubber band $(s=0)$ was as follows: $\kappa \sim t^{-1 / 2}$. This research has guiding significance for the design of rubber bands as elastic energy storage devices.
\end{abstract}

Keywords: ejection rubber band, elastodynamics, bending effect, hyperelastic materials, maple

\section{INTRODUCTION}

Stretching, ejection, and retraction of objects are ubiquitous, including the retraction of flat elastic belts ${ }^{[1]}$, the release of catapults ${ }^{[2]}$, rubber band catapults, large catapults in amusement parks, and the strings of some musical instruments. Rubber band ejection is a familiar and interesting experience. The rubber band is stretched and released, and the whole movement process very fast. It is difficult to observe the retracted deformation state of the rubber band during the ejection process. However, it is amazing that the ejection of the stretched rubber band rarely hits the finger. Oratis and Bird ${ }^{[3]}$ performed an experimental and theoretical study of two self similar retractions during rubber band ejection and explained the reason that the rubber band does not hit the hand. At the beginning of the stretching and ejection process, elastic retraction of the rubber band occurs. After the retraction to the initial state without strain, the rubber band as a whole moves forward at a uniform speed due to inertia. The finger can move out of the path of the rubber band during rebound so that it does not get hit by the rubber band. However, the dynamics are complicated.

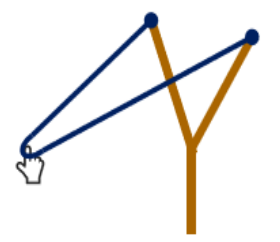

FIG. 1: Slingshot ejection model

Corresponding Author: Bohua Sun.

Email: sunbohua@xauat.edu.cn, http://imt.xauat.edu.cn.
In the study of elastic retraction due to tension after release, the traditional unidirectional springback model only considers the effect of tension and inertia ${ }^{[4,5]}$, but the bending moment is very important in regions with high rates of bending ${ }^{[6,7]}$. When the front end of the rubber band begins to move, it bends dynamically in a manner similar to that of a straight beam or bar ${ }^{[8,9]}$. Mason $^{[10]}$ performed a detailed experimental study of the free contraction that occurs when one end of a stretched rubber band is released. Vermorel et al. ${ }^{[1]}$ studied the dynamic instability of the elastic retraction by tension after release for flat rubber bands. The results of the study confirmed the existence and importance of longitudinal stress waves in the process of the tensile retraction of elastomers. Wegner et al. ${ }^{[1,12]}$ studied the dynamic response after the sudden release of stretched hyperelastic strings. Finite element simulations were used by Oratis and Bird ${ }^{[3]}$, and the results showed that in the process of the rubber band ejection and retraction, rubber bands are under tensile strain $\epsilon$. The corresponding half angle of the wedge angle is $\phi$, and the initial length is $(\epsilon+1) \ell_{0}$, $\ell_{0}$. When the rubber band is released at $t=0$, the rubber band after material point in $V$ retraction of speed, the back-end can form a growing bending area. Oratis and Bird ${ }^{[3]}$ showed through experiments and theoretical analysis that when the influence of the bending effect is ignored in the process of elastic band pop-back shrinkage, the following elastic band springback speed relation is obtained: $\frac{V}{C}=\left(\frac{\epsilon}{\epsilon+1}\right)\left(\frac{1}{1-\sin \phi}\right)$.

The process of rubber band stretching and ejection is very complex, and it is meaningful to analyze the dynamic process. Thus, Section II of this paper begins with a mathematical model of the dynamics of rubber band stretching and ejection, and the dynamic process is comprehensively analyzed. In Section III, the finite element software ABAQUS is used to verify the exper- 
imental results of Oratis and Bird ${ }^{[3]}$ to ensure that the simulation results and data are correct and to verify the relationship between the curvature in the mathematical model and the arc length and time. Based on the results of dimensional analysis, simulations of the stretched ejection of rubber bands with different thicknesses were conducted considering the influence of the effect of bending. Section IV draws conclusions.

\section{MATHEMATICAL MODEL OF STRETCHED RUBBER BAND EJECTION DYNAMICS}

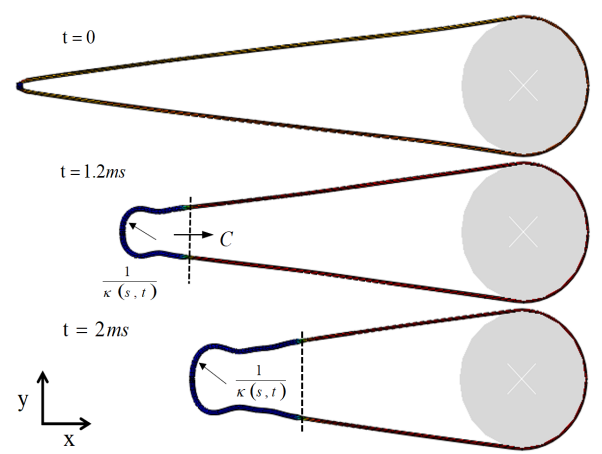

FIG. 2: Rubber band stretching and ejection diagram

During the springback process of rubber bands, a ben$\mathrm{t}$ region with increasing size will form at the back, as shown in Figure 1. Oratis and Bird ${ }^{[3]}$ used a beam model to study the self-similar retraction behavior during rubber band ejection. Here, we model the region after the longitudinal wave passes as an constant-length beam based on the Euler-Bernoulli beam theory ${ }^{[13]}$. The beam cross-sectional area is $A$, the cross-sectional moment of inertia is $I$ (rectangular section $I=\frac{1}{12} b h^{3}, b$ is the width, $h$ is the thickness, $I=\frac{1}{4} \pi r^{4}$, and $r$ is the radius). The following relationships are defined: $\frac{\partial x}{\partial s}=\cos \theta(s, t)$, $\frac{\partial y}{\partial s}=\sin \theta(s, t)$, where $\theta(s, t)$ is the angle between the tangent and the $x$-axis. The small deflection vibration equation of the beam ${ }^{[14]}$ is as follows:

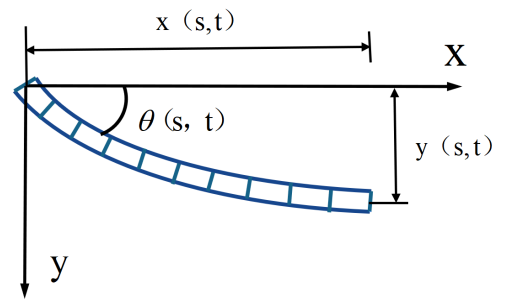

FIG. 3: Beam theory mathematical model of stretched rubber band rebound where $y(s, t)$ is the deflection, $s$ is the arc length, and $t$ is time.

The beam theory model contains the rotation angle $\theta=\frac{\partial y}{\partial s}$ and the curvature $\kappa=\frac{\partial^{2} y}{\partial s^{2}}$. Under the assumption of a small displacement ${ }^{[14]}$, to facilitate the study of the elastic band springback process in this paper, Equation (1) is expressed in the form of a rotation angle $\theta(s, t)$ as follows:

$$
E I \frac{\partial^{4} \theta}{\partial s^{4}}+\rho A \frac{\partial^{2} \theta}{\partial t^{2}}=0
$$

Its self-similar solution is

$$
\begin{aligned}
\theta(s, t) & =C_{1} \frac{s^{2}}{t} \text { hypergeom }\left(\left[\frac{1}{2}, 1\right],\left[\frac{3}{4}, \frac{5}{4}, \frac{3}{2}\right],-\frac{\rho A s^{4}}{64 E I t^{2}}\right) \\
& +C_{2} \text { Fresnels }\left[\frac{1}{\sqrt{2 \pi}}\left(\frac{\rho A}{E I}\right)^{\frac{1}{4}} \frac{s}{\sqrt{t}}\right] \\
& \left.+C_{3} \text { FresnelC }\left[\frac{1}{\sqrt{2 \pi}}\left(\frac{\rho A}{E I}\right)^{\frac{1}{4}} \frac{s}{\sqrt{t}}\right]\right)+C_{4},
\end{aligned}
$$

where $C_{1}, C_{2}, C_{3}$, and $C_{4}$ are unknown parameters. Applying the $\theta(s, t)$ boundary conditions: $\theta(0, t)=\frac{\pi}{2}$, $\theta(s, t \rightarrow 0)=\phi, \frac{\partial^{2} \theta}{\partial s^{2}}(0, t)=0$, and $\frac{\partial^{3} \theta}{\partial s^{3}}(0, t)=0$, we determine that $C_{1}=C_{2}=0, C_{3}=(2 \phi-\pi)$, and $C_{4}=\frac{\pi}{2}$. Thus,

$$
\theta(s, t)=\frac{\pi}{2}+(2 \phi-\pi) \text { FresnelC }\left[\frac{1}{\sqrt{2 \pi}}\left(\frac{\rho A}{E I}\right)^{\frac{1}{4}} \frac{s}{\sqrt{t}}\right]
$$

The material parameters of the rubber band were selected as follows: the radius of the circular section $r=$ $0.8 \mathrm{~mm}$, the diameter of the rubber band $D=64 \mathrm{~mm}$, the density $\rho=1.3 \mathrm{~g} \cdot \mathrm{cm}^{-3}, E=1.1 \mathrm{MPa}$, and the initial wedge half-angle $\phi=11.6^{\circ}$.

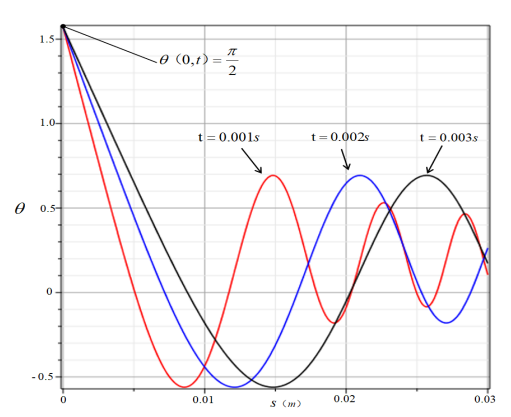

FIG. 4: Variation of $\theta$ with $s$. The curves shown in red, blue, and black correspond to times $t=0.001,0.002$, and $0.003 \mathrm{~s}$. 


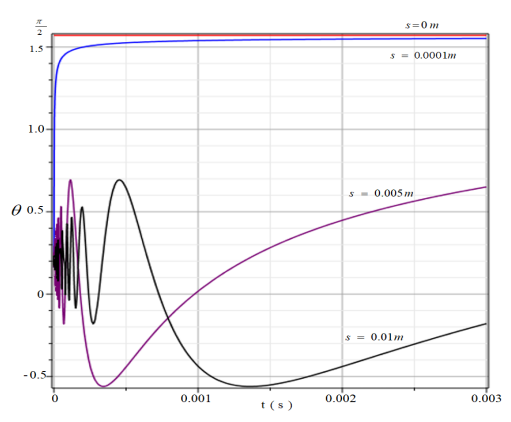

FIG. 5: Variation of $\theta$ with $t$. The arc lengths of the curves shown in red, blue, purple, and black correspond to $s=0$, $0.0001,0.005$, and $0.01 \mathrm{~m}$.

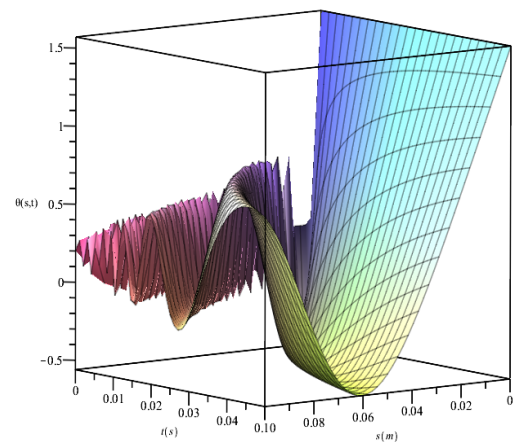

FIG. 6: Three-dimensional phase diagram of $\theta(s, t)$ as a function of time $t$ and arc length $s$.

Fig. 4-6 show the following. (1) When the arc length $s$ is zero, $\theta=\frac{\pi}{2}$. (2) $\theta$ increases with the arc length $s$ during the ejection of the rubber band. It then gradually decreases to zero and then continues to increase in the opposite direction, showing periodic changes. With the increase in the arc length $s, \theta$ changes more drastically. (3) During the rebound of the stretched rubber band in a small arc range, the angle changes smoothly with time. (4) In a short time period after the rubber band is released, the angle changes rapidly, and it finally increases and changes slowly with time.

We define a dimensionless quantity $\xi=$ $s /\left[\left(E I t^{2}\right) /(\rho A)\right]^{1 / 4}$. Thus,

$$
\theta(\xi)=\frac{\pi}{2}+(2 \phi-\pi) \text { FresnelC }\left[\frac{\xi}{\sqrt{2 \pi}}\right]
$$

According to Equations (4) and (5), we can define the complementary angle of $\theta(s, t)$ as $\varphi(\xi)$, that is, $\varphi(\xi)=$ $\pi / 2-\theta(s, t)$. We then have the following: $\varphi(\xi)=(\pi-$ $2 \phi)$ Fresne1C $\left(\frac{\xi}{\sqrt{2 \pi}}\right)$. This differential equation is the same as that obtained by Oratis and Bird when modeling two self-similar retractions of a tensile elastic wedge ${ }^{[4]}$. $\varphi(\xi)$ is obtained by taking the first derivative of $\xi$, as follows: $\varphi^{\prime}(\xi)=\frac{(\pi-2 \phi) \cos \left(\frac{\xi^{2}}{4}\right)}{\sqrt{\pi}}$, When $\varphi^{\prime}(\xi)=0$ and $\varphi(\xi)$ have extreme values, $\xi=\sqrt{2(2 n+1) \pi}, \quad n=0, \ldots$

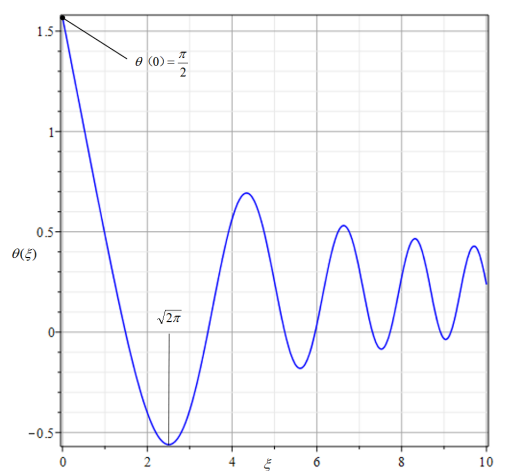

(a)

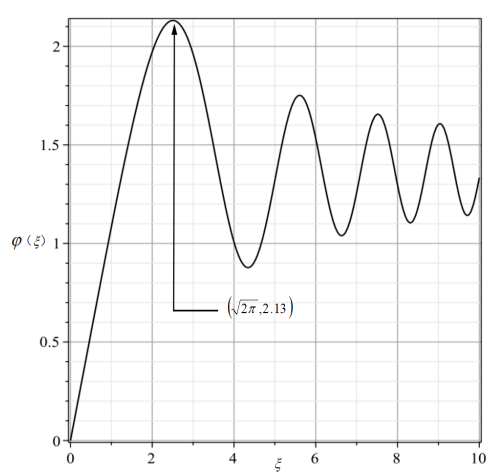

(b)

FIG. 7: (a) Variation of $\theta(\xi)$ with $\xi$. (b) Variation of $\varphi(\xi)$ with $\xi$.

The analysis showed that when $\xi=\sqrt{2 \pi}$, the complimentary angle $\varphi(\xi)$ had a maximum value, at which time the rubber band stretched and retracted to the initial state without strain. As the variable $\xi$ increased gradually, the degree of change in the angle of rotation tended to be gentle. Before the maximum supplementary angle appeared, the recovery process of the elastic energy of the rubber band occurred, after which the rubber band moved in the ejection direction overall.

Combined with Euler-Bernoulli beam theory ${ }^{[14]}$, the curvature $\kappa$ in the process of stretching and springback of rubber band is obtained by Equation (4) as follows:

$$
\begin{aligned}
\kappa(s, t) & =\frac{\partial \theta_{(s, t)}}{\partial s} \\
& =\frac{(2 \phi-\pi)}{\sqrt{2 \pi}}\left(\frac{\rho A}{E I}\right)^{\frac{1}{4}} t^{\frac{1}{2}} \cos \left[\frac{1}{4}\left(\frac{\rho A}{E I}\right)^{\frac{1}{2}} \frac{s^{2}}{t}\right] .
\end{aligned}
$$

When the dimensionless quantity $\xi=$ $s /\left[\left(E I t^{2}\right) /(\rho A)\right]^{1 / 4}$ and the complementary angle $\varphi(\xi)$ takes the maximum value, $\xi=\sqrt{2 \pi}$. Substituting 
this result into Equation (6), $\kappa=0$ when the supplementary angle has the maximum value during the rebound of the rubber band, i.e., the curvature is zero. At this time, there is no bending moment, and the rubber band elastically retracts to its unstretched initial state.

At the origin, where $s=0$, we have the following:

$$
\kappa=\frac{(2 \phi-\pi)}{\sqrt{2 \pi}}\left(\frac{\rho A}{E I}\right)^{\frac{1}{4}} t^{-\frac{1}{2}}
$$

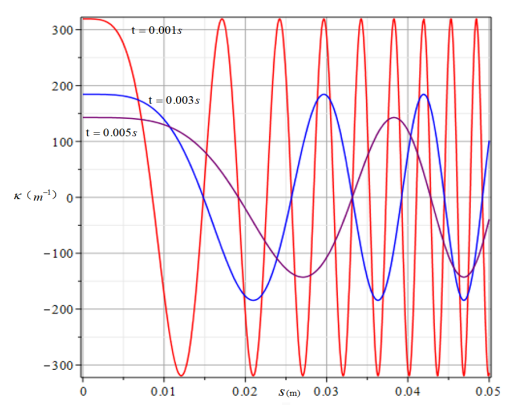

(a)

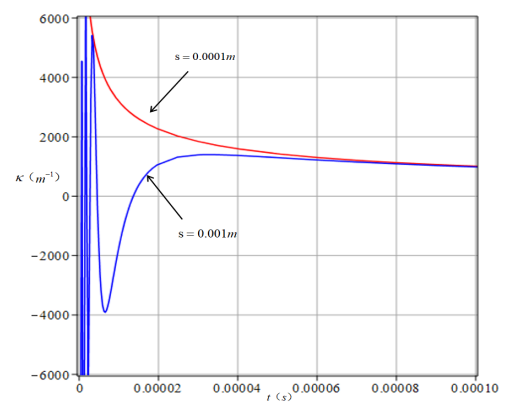

(b)

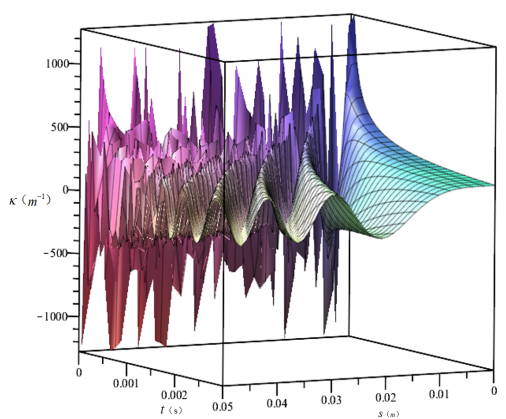

(c)

FIG. 8: (a) Variation of curvature $\kappa$ with arc length $s$ (curvature is positive). The curves shown in red, blue, and purple correspond to times $t=0.001,0.003$, and $0.005 \mathrm{~s}$. (b) Variation of $\kappa$ with time $t$ (curvature is positive). Curves shown in red and blue correspond to arc lengths of $s=0.0001$ and $0.001 \mathrm{~m}$. (c) Phase diagram of curvature $\kappa(s, t)$ as a function of time $t$ and arc length $s$ (curvature is positive).

Fig. 8 shows the following: (1) the curvature changes more sharply with the increase in the arc length, and the fluctuation range of the curvature decreases with the increase in time when the arc length is constant; (2) within a small range of time $t$ after release, the curvature changes dramatically during the springback process and tends to be flat as time increases; (3) there is a non-zero curvature at the origin $(s=0)$, that is, there is an initial curvature at the origin and the curvature is the maximum curvature; with the increase in time, the curvature at the origin gradually becomes smaller and finally decreases to zero, which is also consistent with the phenomenon observed in the simulation.

The differential equation for the deflection of the beam during the rebound process after rubber band ejection can be obtained. The moment in the beam is $M=$ $-E I \frac{\partial \theta}{\partial s}$, which is expressed as follows:

$$
M(s, t)=\frac{(\pi-2 \phi)(\rho A)^{\frac{1}{4}}(E I)^{\frac{3}{4}} t^{-\frac{1}{2}}}{\sqrt{2 \pi}} \cos \left[\frac{1}{4}\left(\frac{\rho A}{E I}\right)^{\frac{1}{2}} \frac{s^{2}}{t}\right]
$$

According to Equations (6) and (8), the curvature and bending moment of the bending region have the same relationship with the arc length and time. There is only one constant difference between the expressions of the curvature and bending moment, and this constant depends on the cross section geometry and material parameters. Similarly, there is an initial bending moment at the origin, and the bending moment changes sharply with the increase in the arc length $s$. In a short period of time after the rubber band is released, the bending moment changes very sharply, and the change tends to be gentle with the increase in time.

The shear force in beam is $Q=-\frac{\partial M(s, t)}{\partial s}$. Thus,

$$
Q(s, t)=\frac{(2 \phi-\pi)(\rho A)^{\frac{3}{4}}(E I)^{\frac{1}{4}} s t^{-\frac{3}{2}}}{2 \sqrt{2 \pi}} \sin \left[\frac{1}{4}\left(\frac{\rho A}{E I}\right)^{\frac{1}{2}} \frac{s^{2}}{t}\right]
$$

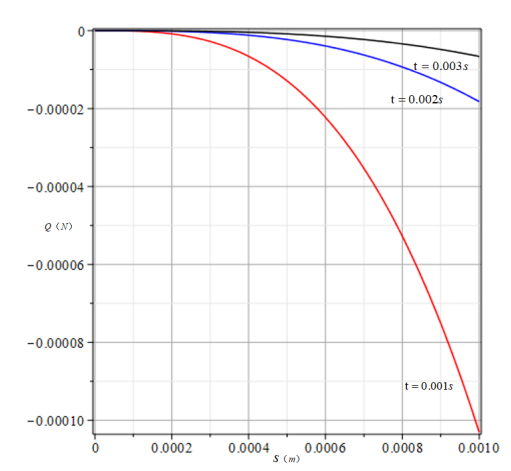

FIG. 9: Variation of shear force $Q$ with arc length $s$. The curves shown in red, blue, and black correspond to times $t=$ $0.001,0.002$, and $0.003 s$. 


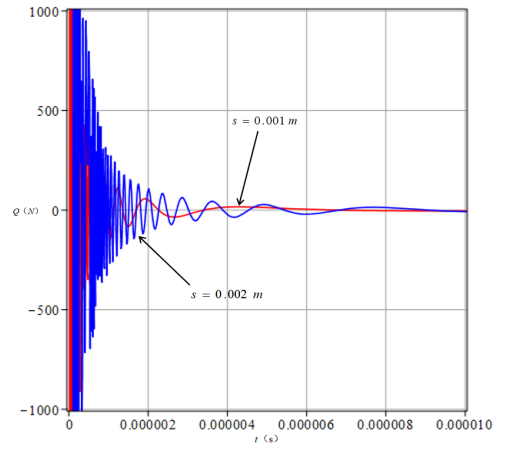

FIG. 10: Variation of shear force $Q$ with time $t$. Curves shown in red and blue correspond to arc lengths of $s=0.001$ and $0.002 \mathrm{~m}$.

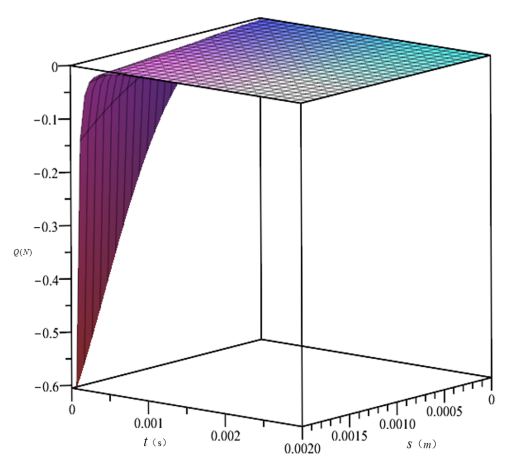

FIG. 11: Three-dimensional phase diagram of shear force $Q$ as a function of arc length $s$ and time $t$.

Fig. (9)-(11) show that (1) the shear force $Q$ is zero at the origin, which satisfies a symmetry condition during elastic ejection, (2) the shear force $Q$ increases gradually with the arc length $s$, and it increases faster and faster, and (3) the shear force changes sharply over a short time, whereas the arc length shear force decreases gradually to zero with time.

\section{FINITE ELEMENT SIMULATION OF RUBBER BAND STRETCHING EJECTION DYNAMICS}

We used the material and size of the rubber band used by Oratis and Bird ${ }^{[3]}$ to verify the experimental and theoretical results and to ensure the feasibility of the finite element model and the accuracy of the extracted data.

\section{Extraction of experimental parameters of superelastic materials}

In this paper, the experimental data, the stress-strain curve of the rubber band material (Fig. 12), and the material parameters reported by Oratis and Bird ${ }^{[3]}$ were used in the simulation (Table).

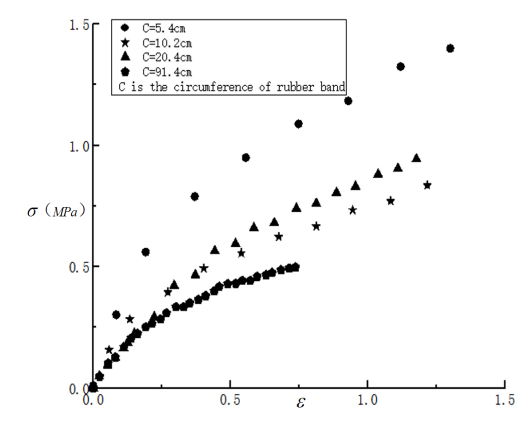

FIG. 12: Stress-strain curves of various kinds of rubber bands. The constitutive parameters of the rubber bands were obtained from the report of Oratis and Bird ${ }^{[3]}$ on rubber band ejection.

A rubber band is a hyperelastic material ${ }^{[15-21]}$. Based on the assumption that isotropy and volume are approximately incompressible, a variable-density function is usually used to characterize rubber. At present, the polynomial strain energy function ${ }^{[22]}$ (generalized Mooney-Revlin function), which is widely used in finite element analysis, is expressed as follows: $U=$ $\sum_{i+j=N}^{N} C_{i j}\left(I_{1}-3\right)^{i}\left(I_{2}-3\right)^{j}+\sum_{k=1}^{N} \frac{1}{d_{k}}\left(\sqrt{I_{3}}-1\right)^{2 k}$, where $N$ is the order of the function, $C_{i j}$ and $d_{k}$ are material constants, which are usually obtained by experiments, and $I_{1}, I_{2}$, and $I_{3}$ are strain invariants of order 1,2 , and 3 , respectively.

The finite element software ABAQUS was used to analyze the experimental material data, and the two-parameter Mooney-Revlin model ${ }^{[23,24]}, \quad W=$ $C_{10}\left(I_{1}-3\right)+C_{01}\left(I_{2}-3\right)$, was used to study the elastic band material, as shown in Fig. 12, because it has better stability.

\begin{tabular}{|c|c|c|c|c|c|}
\hline circumference & $C(\mathrm{~cm})$ & 5.4 & 10.2 & 20.4 & 91.4 \\
\hline width & $b(\mathrm{~mm})$ & 1.2 & 13 & 1.6 & 25.4 \\
\hline thickness & $h(\mathrm{~mm})$ & 1.1 & 1.7 & 2.0 & 1.7 \\
\hline density & $\rho\left(\mathrm{gcm}^{-3}\right)$ & 0.8 & 1.2 & 1.3 & 1.1 \\
\hline modulus of elasticity & $E(\mathrm{MPa})$ & 1.5 & 1.1 & 1.1 & 1.1 \\
\hline coefficient & $C_{10}$ & 0.069 & 0.203 & 0.187 & -0.024 \\
\hline coefficient & $C_{01}$ & 0.586 & 0.046 & 0.122 & 0.34 \\
\hline
\end{tabular}

Table. Dimensions and material parameters of rubber bands with rectangular cross-sections were obtained from the report of Oratis and Bird ${ }^{[3]}$ on rubber band ejection. A circular cross section of the rubber band was assumed, and the parameters were as follows: $r=0.8 \mathrm{~mm}, D=$ $64 \mathrm{~mm}, \rho=1.3 \mathrm{~g} \cdot \mathrm{cm}^{-3}, E=1.1 \mathrm{MPa}, \phi=11.6^{\circ}, C_{10}$ $=0.187$, and $C_{01}=0.122$.

\section{Simulation results and verification}

The material parameters for the selected model were as follows: $C=20.4 \mathrm{~cm}, b=1.6 \mathrm{~mm}, h=1.9 \mathrm{~mm}, \rho=1.3 \mathrm{~g}$. $\mathrm{cm}^{-3}, E=1.1 \mathrm{MPa}, \epsilon=0.476, \phi=14^{\circ}, C_{10}=0.187$, 
and $C_{01}=0.122$. Rigid cylindrical contact was specified at the front end of the stretched rubber band, the normal and tangential directions were both hard contacts, and the rubber band was modeled using the solid C3D8R units of the ABAQUS software. The finite element model results for the stretching and ejection of the rubber band with the above material parameters are shown in Fig. 13 (see the attachment for the simulation dynamics results).

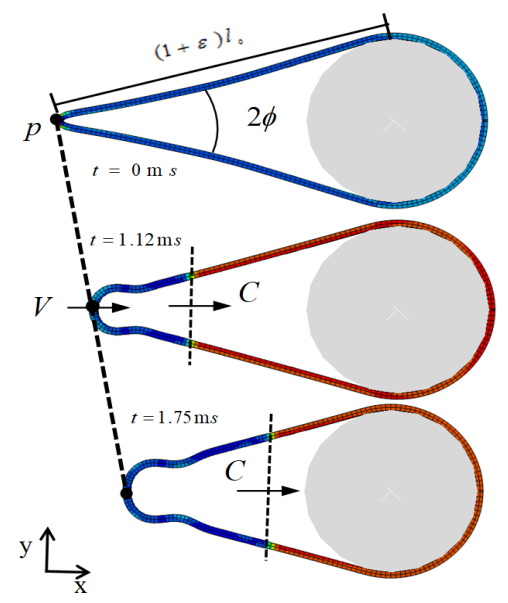

FIG. 13: Stretched rubber band ejection simulation.

Using the finite element model established above, the midpoint $p$ of the end of the rubber band (Fig. 13) was taken as the reference point, and its speed was $V$.

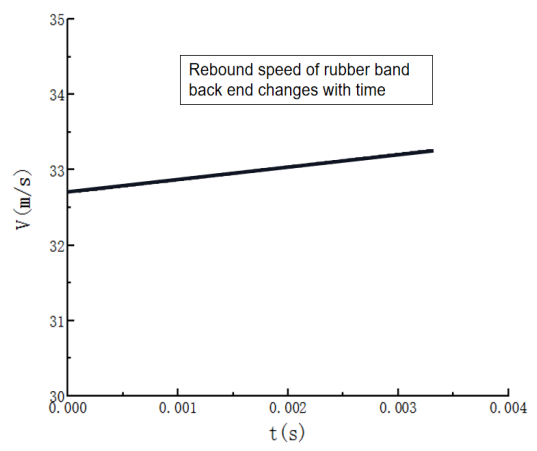

FIG. 14: Rear rebound speed of the rubber band, $V$

The rebound speed $V$ at the back end of rubber band increased with time, but the overall change was small. In fact, after the release of the stretched rubber band, the rubber band began to shrink, and the rear end moved in the ejection direction, which indicated that the elastic recovery of the stretched rubber band was accompanied by the rapid release of elastic energy.

After the elastic recovery process of the rubber band was complete, the whole rubber band moved in the ejection direction due to inertia. According to Newton's second law, $F=m a$, when the external force is $F=0$, the speed of the elastic rebound point is uniform along the ejection direction.

Using the same modeling method, combined with the experimental theory of Oratis and Bird ${ }^{[3]}$, the stretching and ejection of elastic bands with different strain rates, wedge half angles, materials, and sizes were simulated (the results are shown in Fig. 15).

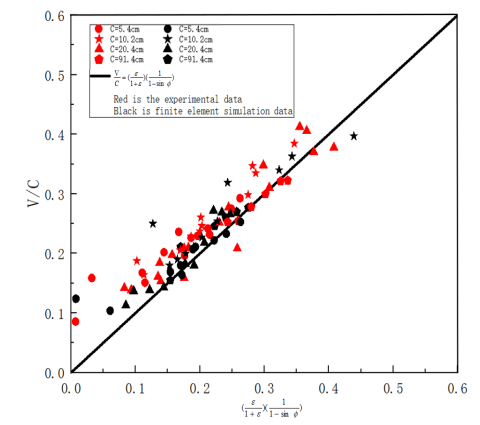

FIG. 15: Experimental results of Oratis and Bird ${ }^{[3]}$ and the results of the finite element simulation in this paper (rectangular cross-sections).

In Fig. 15, the red points are the experimental data of Oratis and Bird ${ }^{[3]}$, and the black points are the simulation results of this work. The results were consistent with each other, and the difference between them was small, indicating the accuracy of the simulation and extracted data in this work. The theoretical results obtained by Oratis and Bird ${ }^{[3]}$ ignored the bending effect, and these results were different than the simulation results in this paper, indicating that the influence of the bending effect on this dynamic process must be further discussed.

Based on the elastic stretching dynamics model of the ejection process, we examined the motion of the elastic rebound endpoint $(s=0)$. The curvature is expressed as follows: $\kappa=\frac{2 \phi-\pi}{\sqrt{2 \pi}}\left(\frac{\rho A}{E I}\right)^{\frac{1}{4}} t^{-\frac{1}{2}}$.

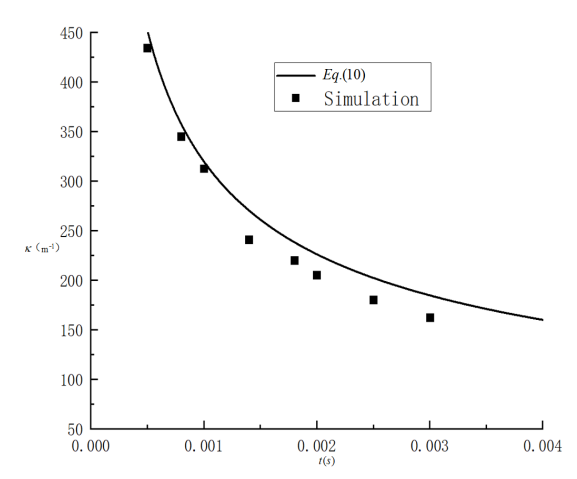

FIG. 16: Variation of curvature $\kappa$ with time $t$ at the end of the elastic during retraction (curvature is positive).

Fig. 16 shows the simulation results of this paper (the model parameters are shown in Table ). The solid line 
represents the theoretical results obtained by introducing the beam mathematical model. The curvature of the end of the rubber band during the retraction process decreased with time and finally reached zero, with a decreasing trend of $\kappa \sim t^{-\frac{1}{2}}$. This agrees with the theoretical relationship given by Equation (7) and verifies the accuracy of the mathematical model of the stretched rubber band ejection dynamics.

\section{Influence of bending effect on tensile ejection dynamics}

Through dimensional analysis (see the appendix), we determine the equation for the speed of the rebound process: $\frac{v}{c}=f\left(\epsilon, \phi, \frac{h}{b}\right)$.

In the simulation process, we can directly see the change of the bending area in the springback process of the rubber band. We selected the simulation result$\mathrm{s}$ of the model shown in Fig. 13, where $\epsilon=0.476$ and $\phi=14^{\circ}$. We ignored the influence of bending effect (Fig. 17).

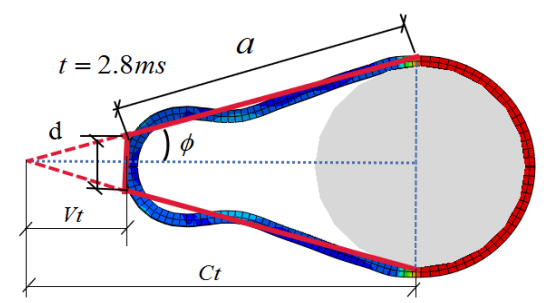

FIG. 17: During the rebound process of the rubber band, the influence of the bending effect is ignored

When the longitudinal wave reaches the cylinder, the position of the longitudinal wave is $c t=(1+\epsilon) \ell_{0} \cos \phi$, and the sum of half the length of the upper side and one leg of the trapezoid was approximately equal to $\ell_{0}$, that is, $\ell_{0}=d / 2+a$. $a$ and $d$ are the lengths of trapezoid leg and the upper and lower sides, respectively. The geometric relationship $c t \tan \phi-a \sin \phi=d / 2$ yields the following: $a=\frac{1-(1+\varepsilon) \sin \phi}{1-\sin \phi} l_{0}, d=\frac{2 \varepsilon l_{0} \phi}{1-\sin \phi}$. The geometric relationship is $\frac{V}{C}=\frac{d}{2} /(c t \cdot \tan \phi)$. Therefore, $\frac{V}{C}=\left(\frac{\epsilon}{1+\epsilon}\right)\left(\frac{1}{1-\sin \phi}\right)$. According to this equation, the dynamics equation obtained by ignoring the influence of the bending effect on the springback dynamics of the rubber band (which is independent of the cross-sectional shape of rubber band at this time) is consistent with the result of Oratis and Bird ${ }^{[3]}$. At the same time, it was proven that the dimensional analysis of the elastic rebound dynamics was correct.

Considering the influence of the bending effect on the dynamics of the whole retraction process, the dynamic relationship between the velocity $V$ and the longitudinal wave velocity $C$ is as follows: $\frac{V}{C}=f\left(\epsilon, \phi, \frac{h}{b}\right)$. To en- sure that the strain $\epsilon$, wedge half angle $\phi$, and rubber band width $b$ are fixed values, rubber bands with different thicknesses $h$ are used for the simulation, and the simulation results are shown in Fig. 18.

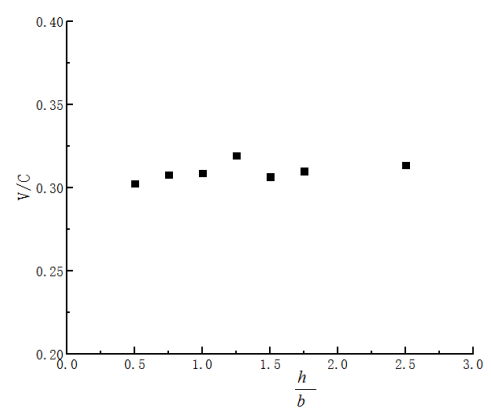

FIG. 18: Influence of thickness $h$ on the springback dynamics

As shown in Fig. 18, during the springback process of rubber bands with different thicknesses $h$, the thickness of the rubber band has little influence on the springback dynamics. This is mainly because the thickness $h$ is smaller than the diameter of the rubber band and the main stretching length scale, and the influence of the thickness $h$ on the elastic dynamics is not significant. It is speculated that rubber bands with different cross-sectional shapes exhibit the same dynamics during stretching ejection when considering the bending term. The rubber band is stretched until the wedge half angle is $\phi$ and has strain $\epsilon$. The tensile stress inside the rubber band is dominant when stretching and retracting, and the bending effect is small at this time. When the rubber band is retracted to a certain extent, the influence of the tensile stress inside the rubber band is significantly reduced, and the influence of bending gradually increases. Thus, the influence of the bending tensile ejection dynamics cannot be ignored.

Considering the influence of the bending of the rubber band during springback, the experimental results of $\mathrm{O}$ ratis and $\operatorname{Bird}^{[3]}$ and the simulation results in this paper were fitted linearly, as shown in Fig. 19.

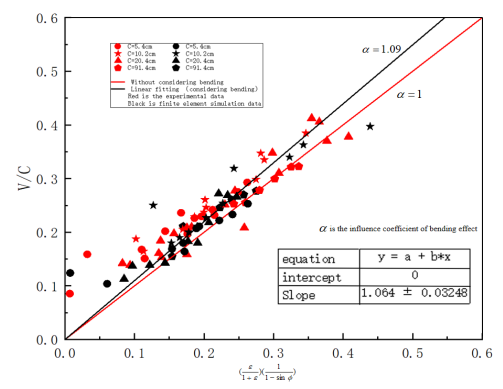

FIG. 19: Linear fit of experimental (Oratis and Bird ${ }^{[3]}$ ) and the simulation results in this paper (rectangular crosssections).

There was significant difference between the two re- 
sults. Therefore, considering the influence of the bending area on the ejection of the stretched rubber band, the coefficient $\alpha$ is introduced to modify the springback velocity relation as follows:

$$
\frac{V}{C}=\alpha\left(\frac{\epsilon}{\epsilon+1}\right)\left(\frac{1}{1-\sin \phi}\right),
$$

where $\alpha$ was determined by fitting to be 1.09 .

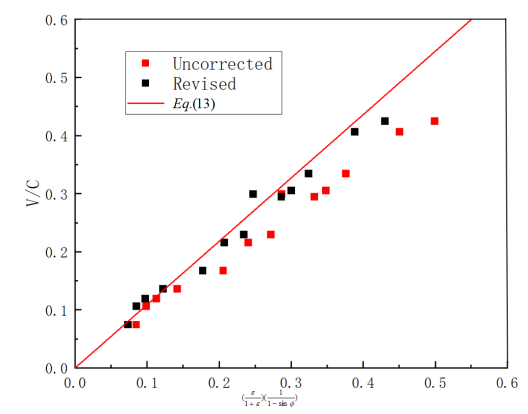

FIG. 20: Simulation results for circular sections with and without bending.

Based on a previous study, the cross-section was substituted for simulation, and a circular cross-section (Table ) was used to simulate the results (Fig. 20). The fitting degree of the theoretical and finite element simulation results were improved by considering the correction factor of the bending moment. When the cross-sectional dimensions of the rubber band were small compared with the main length scale, the influence of the cross-sectional shape and size on the elastomer stretching and ejection velocity can be ignored within a certain range of requirements.

\section{CONCLUSIONS}

The conclusions of this research are as follows:

(1) A simple Euler-Bernoulli beam model was used to analyze the stretched elastic band ejection process. The results showed that the simple beam model of the dynamics can describe the whole ejection process well.

(2) The bending area changed constantly during the rebound of the rubber band. According to the mathematical model of this paper, the curvature relationship is given by Equation (6). Combined with the finite element simulation results, the results at the origin were verified and fit well with experimental data. In addition, the curvature value at the origin of the rubber band was the largest in the process of stretching and ejection, and it had an initial curvature that was not zero. The variation of the curvature at the origin with time $t$ and arc length $s$ is given by Equation (7).

(3) By solving and analyzing the mathematical model of the stretched rubber band ejection dynamics, we determined that the angle $\theta$, curvature $\kappa$, bending moment
$M$, and shear force $Q$ change sharply in a short initial period during the ejection. This also agreed with the fact that a large amount of energy is released rapidly at the moment of ejection, resulting in significant springback deformation.

(4) The factors that affect the rebound velocity relationship $\frac{V}{C}$ in the process of stretching and ejection can be determined by dimensional analysis. The simulations presented in this paper were in good agreement with the experimental results of Oratis and Bird, which proved the accuracy of the simulation and data extraction. Without considering bending, the theoretical results of Oratis and Bird were consistent with the experimental and simulation results, but there were some differences. In this paper, the influence of the bending effect on the stretched elastic ejection was considered. By changing the crosssectional thickness $h$, which affects the bending moment, and comparing the experimental results of Oratis and Bird and the simulation data in this paper, the correction coefficient of $\alpha=1.09$ to account for bending effect was obtained.

[1] Vermorel, Romain, Nicolas Vandenberghe, and Emmanuel Villermaux. "Rubber band recoil." Proceedings of the Royal Society A: Mathematical, Physical and Engineering Sciences 463.2079 (2007): 641-658.

[2] Hickman, Clarence Nicholas. "The dynamics of a bow and arrow." Journal of Applied Physics 8.6 (1937): 404409.

[3] Oratis, Alexandros T., and James C. Bird. "Shooting rubber bands: Two self-similar retractions for a stretched elastic wedge." Physical review letters 122.1 (2019): 014102.

[4] Gent, A. N., and P. Marteny. "The effect of strain upon the velocity of sound and the velocity of free retraction for natural rubber." Journal of Applied Physics 53.9 (1982): 6069-6075.

[5] Stambaugh, R. B. "The retraction of stretched rubber." Rubber Chemistry and Technology 17.3 (1944): 617-618.

[6] Audoly, Basile, and Sébastien Neukirch. "Fragmentation of rods by cascading cracks: why spaghetti does not break in half." Physical review letters 95.9 (2005): 095505.

[7] Callan-Jones, A. C., P-T. Brun, and B. Audoly. "Selfsimilar curling of a naturally curved elastica." Physical review letters 108.17 (2012): 174302.

[8] Gladden, J. R., et al. "Dynamic buckling and fragmentation in brittle rods." Physical review letters 94.3 (2005): 035503.

[9] Cross R C, Wheatland M S. "Modeling a falling slinky." American Journal of Physics, 80(12) (2012): 1051-1060.

[10] Mason, P. "Finite elastic wave propagation in rubber." Proceedings of the Royal Society of London. Series A. Mathematical and Physical Sciences 272.1350 (1963): $315-330$

[11] Wegner, J. L., J. B. Haddow, and R. J. Tait. "Unloading waves in a plucked hyperelastic string." (1989): 459-465.

[12] Wegner, J. L., and J. B. Haddow. "An experimental s- 
tudy of the unloading waves in a plucked hyperelastic string." (1990): 667-671.

[13] Bauchau, O. A., and J. I. Craig. "Euler-Bernoulli beam theory." Structural analysis. Springer, Dordrecht, 2009. $173-221$.

[14] Audoly, Basile, and Sébastien Neukirch. "Fragmentation of rods by cascading cracks: Why spaghetti does not break in half." Physical review letters 95.9 (2005): 095505.

[15] Peng Xiangfeng, Li Luxian. "State of the art of constitutive relations of hyperelastic materials." Chinese Journal of Theoretical and Applied Mechanics 52(5) (2020): $1221-1232$.

[16] Upadhyay, Kshitiz, Ghatu Subhash, and Douglas Spearot. "Thermodynamics-based stability criteria for constitutive equations of isotropic hyperelastic solids." Journal of the Mechanics and Physics of Solids 124 (2019): 115-142.

[17] Bingdong, Tan, et al. "A transversely isotropic viscohyperelastic constitutive model for short fiber reinforced EPDM." Chinese Journal of Theoretical and Applied Mechanics 49.3 (2017): 677.

[18] Li Xiaofang, Yang Xiaoxiang. "Hyperelastic constitutive models of rubber materials." China Elastomerics 1 (2005): 52-60.

[19] Bingdong, Tan, et al. "Hyperelastic constitutive model for short fiber reinforced EPDM inhibitor film." Chinese Journal of Theoretical and Applied Mechanics 49.2 (2017): 317

[20] Kshitiz U, Ghatu S, Douglas S. "Thermodynamics-based stability criteria for constitutive equations of isotropic hyperelastic solids." Journal of the Mechanics and Physics of Solids 124 (2019): 115-142.

[21] Zhang Xirun, Cai Lixun, Chen Hui. "Hyperelastic indentation models and the dualindentation method based on energy density equivalence." Chinese Journal of Theoretical and Applied Mechanics 52(3) (2020): 787-796.

[22] Seibert, D. J., and N. Schoche. "Direct comparison of some recent rubber elasticity models." Rubber chemistry and technology 73.2 (2000): 366-384.

[23] Rivlin, R. S. "Large elastic deformations of isotropic materials IV. Further developments of the general theory." Philosophical Transactions of the Royal Society of London. Series A, Mathematical and Physical Sciences 241.835 (1948): 379-397.

[24] Mooney, Melvin. "A theory of large elastic deformation." Journal of Applied Physics 11.9 (1940): 582-592.

[25] B. H. Sun, "Dimensional Analysis and Lie Group." (China High Education Press,Beijing, 2016).

\section{Appendix}

Dimensional analysis of rubber band stretch ejection dynamics:
Based on dimensional analysis theory ${ }^{[25]}$, seven parameters were determined (see the table below), among which there are three basic dimensions: L, M, and T.

\begin{tabular}{lll}
\hline variable & symbol & dimension \\
\hline width & $\mathrm{b}$ & $\mathrm{L}$ \\
thick & $\mathrm{h}$ & $\mathrm{L}$ \\
density & $\rho$ & $M L^{-3}$ \\
elastic Modulus & $\mathrm{E}$ & $M L^{-1} T^{-2}$ \\
strain & $\epsilon$ & 1 \\
wedge half angle & $\phi$ & 1 \\
speed & $V$ & $L T^{-1}$ \\
\hline
\end{tabular}

Table Dimensions of physical quantities.

Thus, this problem produces $7-3=4$ dimensionless quantities $\Pi$, shown as follows:

$$
\Pi_{1}=\epsilon, \quad \Pi_{2}=\phi, \quad \Pi_{3}=\frac{h}{b}, \quad \Pi_{4}=E \rho^{a} V^{b} h^{c},
$$

where a, b, and c can be solved by dimensionless conditions;

$$
\begin{aligned}
\operatorname{dim}\left(\Pi_{4}\right) & =M L^{-1} T^{-2} M^{a} L^{-3 a} L^{b} T^{-b} L^{c} \\
& =M^{1+a} L^{b-3 a+c-1} T^{-2-b},
\end{aligned}
$$

The following must be satisfied: $1+a=0, b-3 a+c-1=$ 0 , and $-2-b=0$. Thus, $a=-1, b=-2$, and $c=0$. The dimensionless quantities can be obtained by introducing the determined $\mathrm{a}, \mathrm{b}$ and $\mathrm{c}$ into $\Pi_{4}$ :

$$
\Pi_{4}=\frac{E}{\rho V^{2}}
$$

In this formula, $E$ represents the elastic modulus, $\rho$ represents the density, and $V$ represents the speed at which the rubber band springs back to the material point. Substituting $C=\sqrt{E / \rho}$ into the above equation to modify the dimensionless quantity $\Pi_{4}$, we obtain the following: $\Pi_{4}=\frac{V}{C}$.

According to the theory of dimensional analysis, $\Pi_{4}=$ $f\left(\Pi_{1}, \Pi_{2}, \Pi_{3}\right)$, which can be written as follows:

$$
\frac{V}{C}=f\left(\epsilon, \phi, \frac{h}{b}\right)
$$

Thus, the ejection speed of the rubber band stretching is related to the strain $\epsilon$, initial wedge half angle $\phi$, and bending effect. The specific relationship must be determined by experiments. 